\title{
Plans for Utilizing the Keck Outrigger Telescopes at NPOI
}

\author{
Michael DiVittorio* ${ }^{a}$, Donald J. Hutter ${ }^{a}$, Michael Kelley ${ }^{b}$ \\ ${ }^{a}$ US Naval Observatory Flagstaff Station, 10391 W. Naval Observatory Rd., \\ Flagstaff AZ, 86001, USA \\ ${ }^{\mathrm{b}}$ MHK Technology, 4615 NE Indian River Dr., Jensen Beach FL, 34957, USA
}

\begin{abstract}
Four 1.8 m outrigger telescopes were procured by NASA for use as part of the Keck Observatory Interferometer. Due to changes in NASA planning they will not be installed on Mauna Kea. The Navy Prototype Optical Interferometer (NPOI), a joint project of The U.S. Naval Observatory (USNO), The Naval Research Laboratory (NRL), and Lowell Observatory, located outside Flagstaff, Arizona, would like to upgrade the current siderostats on the project to larger apertures. The preliminary plans for integrating the Keck outrigger telescopes (OT) into NPOI are presented.
\end{abstract}

Keywords: NPOI, optical interferometry, Keck Outriggers

\section{INTRODUCTION}

The NPOI has been in operation since 1996 using $12 \mathrm{~cm}$ apertures on $~ 19-38 \mathrm{~m}$ baselines to perform astrometry. Furthermore, additional $12 \mathrm{~cm}$ apertures can be configured to have baselines up to $437 \mathrm{~m}$ for purposes of imaging. While possessing impressive capabilities, the NPOI is limited to relatively bright objects due to the small apertures used.

In the mid 1990s Keck Observatory, with funding from NASA, had EOST design and build 4, $1.8 \mathrm{~m}$ alt-azimuth telescopes to be used with the Keck interferometer. Due to changes in the NASA interferometry budget, funds are no longer available to install the telescopes and domes on Mauna Kea. While the details of the design and performance of the telescopes are available in earlier SPIE proceedings ${ }^{1,2}$, suffice it to say that the telescopes were designed, when used with additional auxiliary optics, to produce a $\sim 125 \mathrm{~mm}$ diameter beam. This is the same size beam used at NPOI, and with considerable effort the telescopes could be installed for use at NPOI. It appears that NASA would prefer to see the telescopes used at an interferometer, and NPOI is in need of larger apertures, therefore, an agreement is being negotiated between Keck Observatory and the USNO and hopefully Keck will transfer ownership of the telescopes to the USNO.

We present preliminary engineering plans for the installation and integration of the outriggers at NPOI including preliminary budget and schedule.

\section{NPOI: CURRENT CONFIGURATION AND CAPABILITY}

\subsection{NPOI History}

The Navy Prototype Optical Interferometer (NPOI) ${ }^{3}$ has been in operation at the Lowell Observatory site on Anderson Mesa, Arizona, since 1996. An aerial view of the site is shown in Figure 1.

*miked@nofs.navy.mil 


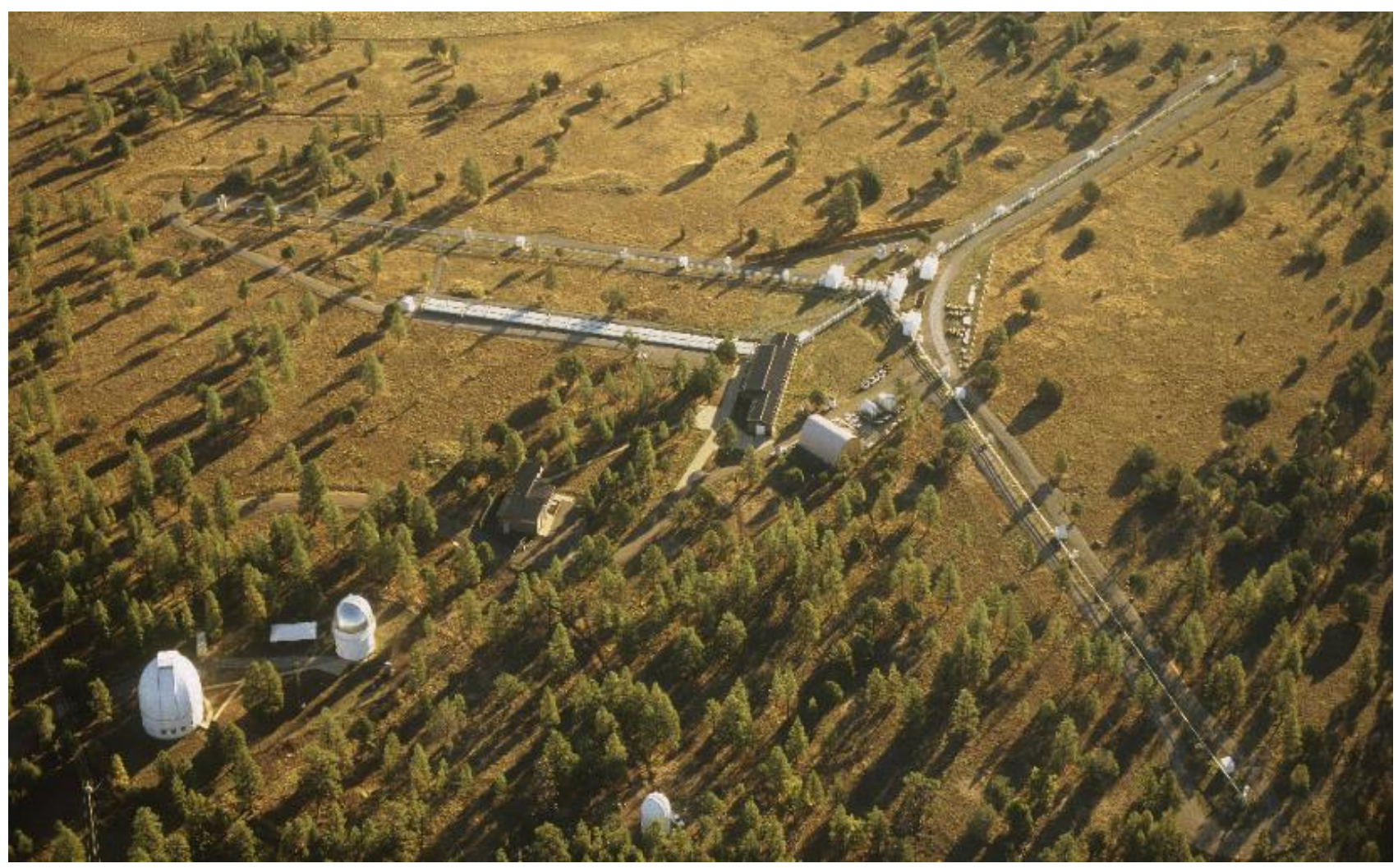

Figure 1. NPOI site, Flagstaff, AZ (Photo courtesy of Michael Collier).

\subsection{Current Configuration}

The interferometer consists of two integrated instruments: an astrometric array and an imaging array; both of which currently share feed and delay lines, and are optically compatible. The wavelength range of operation is in the visible spectrum between $550 \mathrm{~nm}$ and $850 \mathrm{~nm}$. The present array configuration consists of six siderostat stations ${ }^{4}$ : four astrometric in fixed locations and two imaging stations that are moveable along a Y-shaped array. A maximum baseline length of $79 \mathrm{~m}$ can presently be achieved. Light from each siderostat is conveyed through evacuated feed pipes and relay stations to an optics lab, where a periscope lowers the beam and directs it toward a continuously-adjustable fast delay line. The fast delay line provides from 0 to 33 meters of continuously variable optical path. The light then exits the fast delay line and proceeds to the beam-combiner where interference fringes are generated and corresponding data collected for further analysis. The detail of the systems was presented by Armstrong et.al ${ }^{3}$, a short summary of which is presented here.

\subsubsection{Beam Transport Lines}

All light transport paths are in vacuum. This includes the 3 "light pipes" that extend out each of the array arms. One pipe travels the full extent of each arm $(250 \mathrm{~m})$ while the other 2 stop at intermediate distances, stations 8 and $9, \sim 100 \mathrm{~m}$ and $150 \mathrm{~m}$ respectively, along each array arm. Six light pipes travel from the center of the array to the beam combining building, again under vacuum to reduce the affects of atmosphere on the light beams. A total of over $2000 \mathrm{~m} \mathrm{of} 20 \mathrm{~cm}$ vacuum beam transport lines, not including delay lines, are in use at NPOI.

\subsubsection{Fast Delay Lines}

The six fast delay lines (FDL), located in the beam combining building, are based on a JPL design ${ }^{5}$. Each consists of 20 meter long vacuum pipes $41 \mathrm{~cm}$ in diameter. In each pipe is a retro reflector cart which rolls on a rail. Each reflector cart 
is moved by a stepper motor driven auxiliary cart which is voice coil coupled to the main cart. The retro reflector is then positioned by a second voice coil actuator and, finally the flat secondary mirror of the reflector is piezo mounted. The FDLs can track fringes at up to $\sim 50 \mathrm{~Hz}$ with $<20 \mathrm{~nm}$ rms noise while moving at speeds up to $2 \mathrm{~m} / \mathrm{s}$.

\subsubsection{Long Delay Lines}

Each of the six long delay lines (LDL) consist of $\sim 110 \mathrm{~m}$ of $51 \mathrm{~cm}$ vacuum pipe and are located next to the beam combining laboratory building. Static mirrors which provide large delay off-sets for use in support of long baseline observations far from the zenith can "pop up" at 6 different locations along the pipe. Delays range from 0 to $\sim 440 \mathrm{~m}$ in steps of $\sim 30 \mathrm{~m}$ and therefore overlap with the dynamic range of the FDLs. The LDLs are still in commissioning.

\subsubsection{Siderostats}

Fifty $\mathrm{cm}$ diameter siderostat mirrors and mounts are used to feed $12 \mathrm{~cm}$ apertures at both the imaging and astrometric siderostat locations. The siderostats in the 4 astrometric huts are fixed and provide baselines of up to $38 \mathrm{~m}$. The astrometric siderostats use intricate laser metrology to reference each of the 4 units to the local bedrock. This allows for highly accurate absolute astrometric observations. Imaging siderostats can be placed at 10 different locations along each arm. These locations are at distance up to $250 \mathrm{~m}$ from the center of the array and provide baselines of up to $437 \mathrm{~m}$. The imaging stations do not utilize the baseline metrology system; however, the astrometric siderostats can be used with the imaging stations.

\subsubsection{Beam Combining Room}

For added stability, the beam combing room uses the "room within a room" approach. The $12 \mathrm{~cm}$ beams are reduce to $3.5 \mathrm{~cm}$ by off axis beam compressors before arriving at the beam combiner table, where up to 6 beams can be combined. Details of the design and performance of the beam combiner can be found in previous publications $\mathrm{s}^{3,4}$.

\section{PROPOSED OUTRIGGER TELESCOPE CONFIGURATIOIN}

\subsection{Outrigger Telescopes}

As previously noted, the $1.8 \mathrm{~m} \mathrm{f} / 17$ telescopes, shown in figure 2 were designed for use with the Keck Interferometer on Mauna Kea, Hawaii. Details of the telescope design and performance can be found in previous SPIE proceedings ${ }^{1,2}$.

\subsection{Outrigger Layout}

The baseline layout for the outriggers places one unit on each arm of the array, and one near the center. Positions appear in table 1 in meters with the origin being the center of the array, $\mathrm{x}$ to the north, and $\mathrm{y}$ to the east, with coordinates for station 10 on each arm included for reference. These particular positions were chosen to optimize imaging performance. The layout is shown on the topographic site drawing, figure 3.

$\begin{array}{lrr}\quad \text { Station } & \text { X } & \text { Y } \\ \text { North OT (NOT) } & 1.0 & 97.0 \\ \text { East OT (EOT) } & 51.0 & -12.0 \\ \text { West OT (WOT) } & -74.0 & -66.0 \\ \text { Center OT (COT) } & 15.0 & 20.0 \\ \text { North 10 (N10) } & -26.3 & 250.3 \\ \text { East 10 (E10) } & 232.4 & -101.4 \\ \text { West 10 (W10) } & -201.7 & -148.7\end{array}$

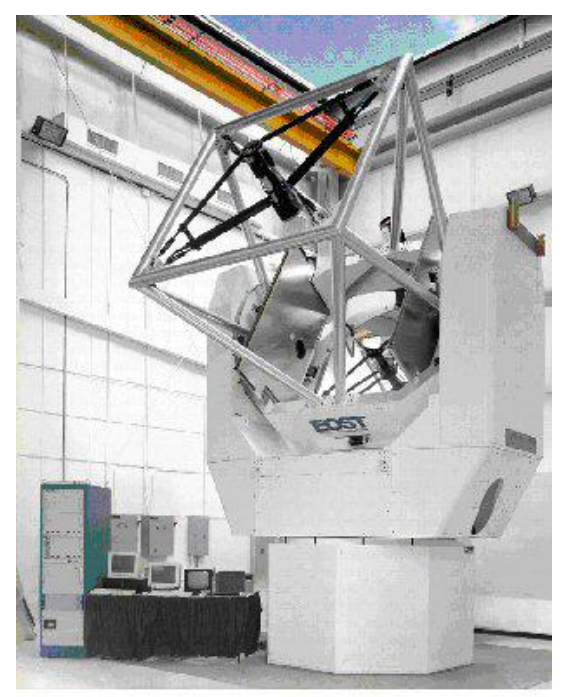

Table 1. Proposed positions of the outrigger telescopes.

Figure 2. Outrigger telescope (Photo courtesy of EOST) 


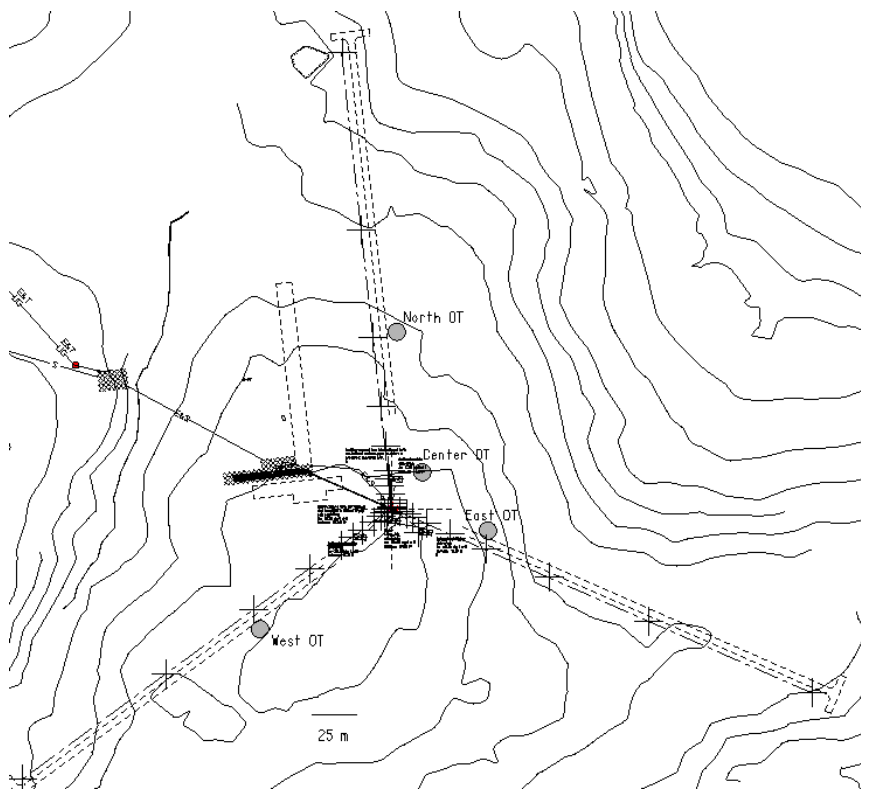

Figure 3. Layout of the outriggers on the NPOI site.

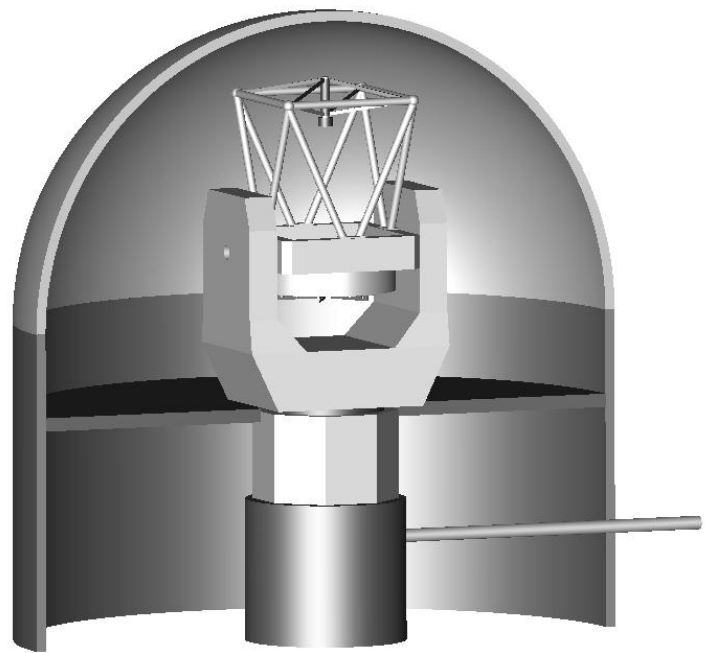

Figure 4. 3D model of outrigger telescope and enclosure.

\subsection{Dome Baseline}

Enclosures were included in the original Keck project, but the USNO has decided to not utilize them. While the enclosures are very nice units, experience from other projects using such enclosures indicated that installation was very expensive and time consuming. Since this installation cost had not already been paid for by Keck/NASA, this would need to be covered by the USNO budget. Preliminary analysis indicates that the use of simple, "off-the-shelf" commercial domes will actually be less costly than installing the pre-built EOS enclosures. For this reason USNO has opted to use a commercial dome on a simple ring wall.

\subsection{Building}

The current baseline uses a simple steel framed and skinned circular building. It will be insulated and include a cooled electronics/computer enclosure on the first floor and a cooled second level observing floor. Figure 4 shows a 3D model of a telescope and dome with $20 \mathrm{~cm}$ light pipe exiting the hollow telescope pier and passing through the building wall.

\subsection{Site Impact}

The visual impact to the site is an important consideration for this project. Figure 5 shows the NPOI site with an artist's conception of the installed outriggers. Keeping the profile low and in step with the current site development and nearby Lowell Observatory telescopes should minimize visual impact. Prior to any installation work at the site, a permit will be obtained from the US Forest Service that will ensure all environmental and culture resources will be preserved. 


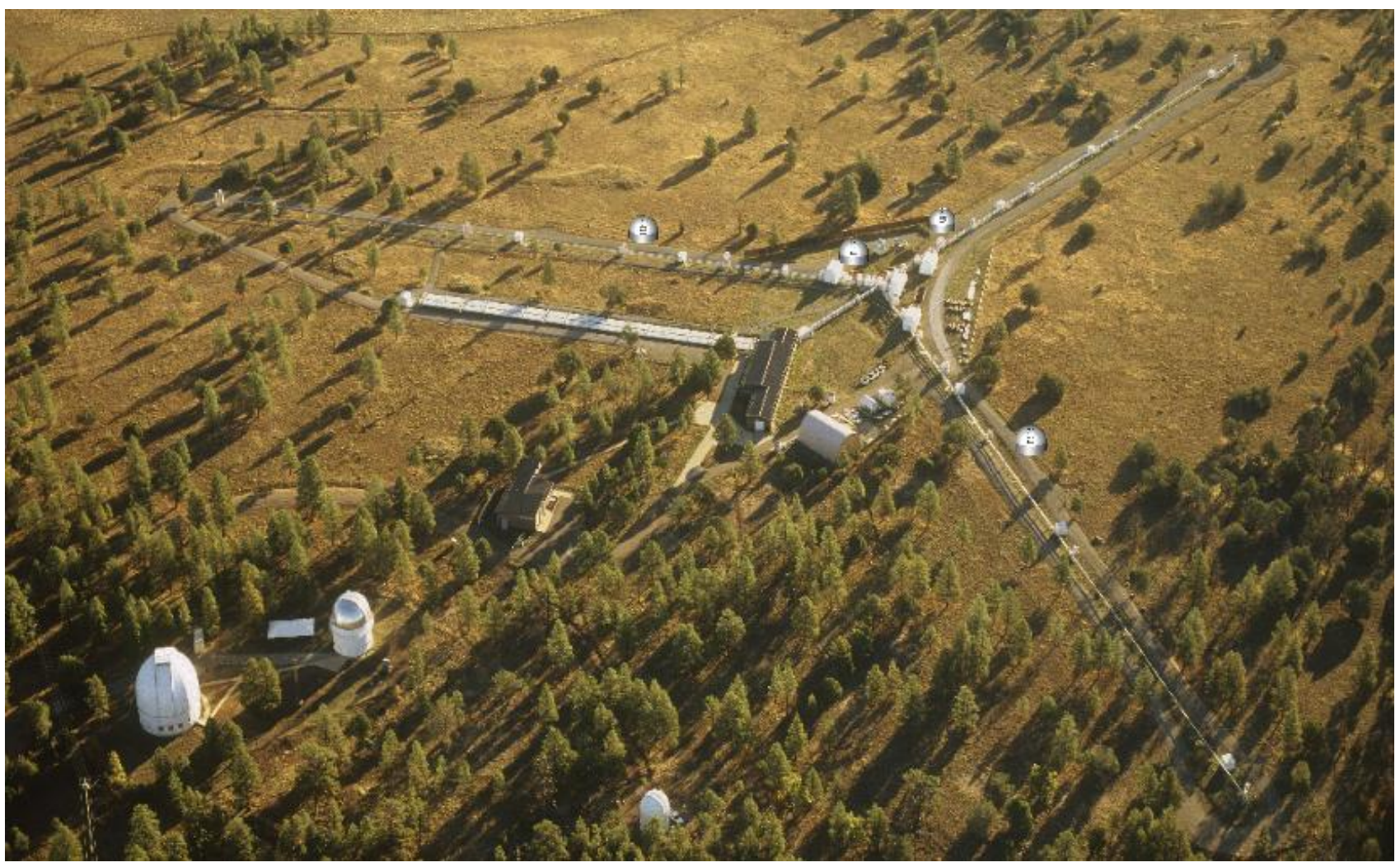

Figure 5. NPOI site with artist conception of outriggers, (Photo courtesy of Michael Collier).

\section{SCIENCE CAPABILITY WITH THE OUTRIGGERS}

NPOI will provide higher angular resolution than current near-infrared interferometers when the longer baselines are implemented. One area of growing interest is measuring the angular size of exoplanet host stars, which can then be used to determine the physical diameter of planets measured transiting their hosts. Utilizing a 180-meter baseline at NPOI (the longest baseline in the nominal OT configuration), observations at 0.7 microns can measure the diameter of a G0 star at 12 pc to $3 \%$. Information of this type can be used to refine photospheric models for relevant stellar types, leading to more precise radii for distant, planet bearing stars. Additionally, NPOI could expand its initial survey of potential TPF target stars for the presence of previously unknown or poorly characterized companions. The OTs would also be very helpful to the wide-angle astrometry program by gaining a couple of magnitudes of sensitivity (easier to do the reference frame tie to the ICRF via observations of radio stars, most of which are $V>6$ ) and with the proposed array configuration offer significantly longer baselines than the current astrometric array that would likely help improve overall astrometric accuracy by a factor of 2-4. Longer baselines and greater sensitivity would help other current NPOI programs such as measuring stellar oblateness for rapid rotators and studying the disks of Be stars.

With the addition of an infrared capability, the NPOI/OT array will enable studies of exoplanets, their host stars, and environments, with unprecedented detail. NPOI/OT will image protoplanets forming in circumstellar disks and will determine the prevalence of Jovian-mass planets around nearby binary stars. Unparalleled advances in observations of circumstellar disks, from planet formation to planet death, will also be achieved as part of a dedicated long-term program. 


\section{ENGINEERING AND INSTALLATION PLANS}

\subsection{Preliminary Engineering plans}

Preliminary engineering plans have been procured for the site/facility work required for installation of the outriggers. Engineering firms were contracted to perform studies of the civil, electrical, and structural requirements for the project. While these are only preliminary plans and are not valid for the actual construction phase, they provide a useful baseline for cost estimation.

\subsubsection{Civil Engineering}

As part of the civil engineering study, a full survey of the proposed locations of the outriggers was performed and tied into the current facility layout. Telescope/pier elevations were identified such that light pipes can feed into the existing light beam transport system from the hollow telescope pier. The realignment of roads and fences as well as the creation of graded staging areas was also addressed.

\subsubsection{Structural Engineering}

The structural engineering study addressed the excavation, pier, building, and dome interface. A "straw-man" building was designed to provide appropriate telescope clearance and height, as well as the specified interface for the commercial off-the-shelf dome. Typical soil conditions and bedrock depth found on the geo-tech report for the original NPOI construction phase were assumed in the plan.

\subsubsection{Electrical Engineering}

While the electrical layout of the outriggers is rather straight forward, understanding the load of each unit and whether or not the current system capacity is adequate is critical. The original project electrical engineer was brought in to analyze the system and determined that that current capacity is adequate for the outrigger upgrade. This is fortunate as a power system upgrade would have represented a significant added expense.

\subsection{Telescope installation}

Telescope installation will follow the procedures developed by EOST for the Mauna Kea installation. EOST may or may not be involved, depending on contractual negotiations. The 3 units currently in storage in Tucson will be shipped to Flagstaff, most likely one at a time, when the facility is ready for installation of each unit. The fourth unit, currently installed and in use in Australia, will remain on loan to EOS until such time that USNO is ready for installation, probably on the order of 2 years from project start. One difference with the NPOI telescope installation plan as compared to the Mauna Kea plan is that the dome is removable which will make for much easier lifts into the enclosure.

\subsection{Control system}

In the last few years NPOI has undertaken a project to develop a new generation of siderostat controller. These are PC based units with motor controller and encoder interfaces, The baseline is to modify this design to control the outriggers. The system will interface to existing outrigger telescope hardware (motor, encoders, etc.) and not to existing outrigger control/interface hardware. Preliminary design of the new interface/control hardware is underway. The method of control for the fast tip-tilt secondary (a Physik Instrumente unit) is still to be determined.

\subsection{Coude path}

The Coude path will roughly follow the plans for the Keck design. The telescope beam will be collimated using an offaxis parabola on an optical bench mounted on the outside of one of the fork arms. Folding flats will steer the beam into the base of the fork, and onto the azimuth axis of the telescope. At this point a $45^{\circ}$ mirror will direct the beam down and 
"off" of the moving part of the telescope. In the hollow masonry pier, another $45^{\circ}$ mirror steers the beam parallel to the ground where it will enter a vacuum transport line and be injected into the existing light transport system. The one major difference from the Keck system is that at NPOI all of the light pipes are above ground, not buried as was the plan for the Mauna Kea installation.

\subsection{Adaptive Optics}

Since the outrigger diameter $(1.8 \mathrm{~m})$ is many times larger than $\mathrm{R}_{\mathrm{o}}(\sim 10 \mathrm{~cm})$ at the NPOI site, adaptive optics will be necessary to utilize the full apertures at anything shorter than mid infrared wave lengths. Therefore, it is assumed that adaptive optics (AO) systems will be implemented on the OTs. Requirements are being defined and preliminary design for the AO system is in work. Since the OTs come with fast tip-tilt secondaries, the AO systems will most likely not include this capability. The decision whether to design/build the systems in house or to procure the systems from a commercial vendor or other scientific institution has not yet been made.

\section{INSTALLATION BUDGET AND SCHEDULE}

\subsection{Estimated Cost Break Down}

Preliminary cost estimates are as follows (for a total of all 4 units):

1) Planning, permitting, and final design;

$0.25 \mathrm{M}$ USD

2) Facility work, including all civil, electrical, and structural (including dome) tasks; $1.5 \mathrm{M}$ USD

3) Telescope installation;

1.0 M USD

4) Control system;

$0.25 \mathrm{M}$ USD

5) Coude path;

0.5 M USD

6) Adaptive optics;

1.5 M USD

Total

$5 \mathrm{M}$ USD

Total $+20 \%$ margin

6M USD

Other than the facility cost, these are very preliminary estimates and are subject to change.

\subsection{Baseline Schedule}

The baseline schedule is a follows, starting when funding is allocated:

Year 1: Permitting and final design.

Year 2: Install facility 1 and telescope 1.

Year 3: Integrate telescope 1 into array, install facilities 2, 3 and 4.

Year 4: Install and integrate telescopes 2, 3 and 4.

Year 5: Install and integrate telescopes 2, 3 and 4 (cont).

Year 6: Install adaptive optics.

\section{SUMMARY}

Negotiations are under way that will hopefully result in the US Naval Observatory taking possession of the Keck Observatory outrigger telescopes. If this happens the telescopes will be installed at the NPOI outside Flagstaff AZ. Utilizing the 4 larger apertures will greatly enhance the science capability of the NPOI and the US interferometry community as a whole. 


\section{ACKNOWLEDGEMENTS}

The Navy Prototype Optical Interferometer is a joint project of the U. S. Naval Observatory and the Naval Research Laboratory, in cooperation with Lowell Observatory, and is funded by the Oceanographer of the Navy and the Office of Naval Research.

\section{REFERENCES}

[1] Craig H. Smith; Bruce H. Cook; Michael Midkiff; Gordon J. Pentland; Robert L. Meeks; James Bell; James M. Walker; Peter L. Wizinowich, "Telescopes for optical/IR interferometry: the NASA/JPL outriggers", Proc. of SPIE, Vol. 5489, pp 720-731, 2004.

[2] James Bell; James M. Walker; Peter L. Wizinowich; Kevin Tsubota; Andy C. Rudeen; Dennis McBride; Kyle K. Kinoshita; Michael Hrynevych; Patricia Goude; M. Mark Colavita; James H. Kelley; Gerard T. van Belle; Robert Brunswick; John K. Little; Craig H. Smith., "Outrigger telescopes for narrow-angle astrometry", Proc. of SPIE, Vol. 5489, pp 962-973, 2004.

[3] J. T. Armstrong, D. Mozurkewich, L. J. Rickard, D. J. Hutter, J. A. Benson, P. F. Bowers, N. M Elias II, C. A. Hummel, K. J. Johnston, D. F. Buscher, J. H. Clark III, L. K. Ha, L.-C. Ling, N. M. White, and R. S. Simon, "The Navy Prototype Optical Interferometer", Astrophysics. J. 496, pp. 550-571, 1998.

[4] C. A. Hummel, J. A. Benson, D. J. Hutter, K. J. Johnston, D. Mozurkewich, J. T. Armstrong, R. B. Hindsley, G. C. Gilbreath, L. J. Rickard, and N. M. White, "First observations with a co-phased six-station optical long-baseline array: application to the triple star eta Virginis", Astrophysics. J. 125, pp. 2630-2644, 2003.

[5] M. Shao, M. M. Colavita, B. E. Hines, D. H. Staelin, D. J. Hutter, K. J. Johnston, D. Mozurkewich, R.S. Simon, J. L. Hershey, J. A. Hughes, and G. H. Kaplan, "The Mark III stellar interferometer", Astronomy and Astrophysics 193, pp. 357-371, 1998. 